THE RING 34 (2012) DOI 10.2478/v10050-012-0002-3

\title{
ACCURACY AND RESOLUTION CAPACITY OF MRL-5(IS) RADAR ORNITHOLOGICAL STATION AND ITS POTENTIAL DEVELOPMENT
}

\author{
Leonid Dinevich and Yossi Leshem
}

\begin{abstract}
Dinevich L., Leshem Y. 2012. Accuracy and resolution capacity of MRL-5(Is) radar ornithological station and its potential development. Ring 34: 3-21.

The paper presents the comprehensive assessment of the resolution capacity of MRL-5(Is) radar ornithological station developed in Israel. Theoretical calculations, as well as experimental testing and field experience enable the authors to evaluate the station's performance and to suggest new directions of its potential development.

The computer-controlled system is able to select bird echoes and plot ornithological charts for distances up to $60 \mathrm{~km}$. Under conditions of normal refraction at distances of $5-25 \mathrm{~km}$, MRL-5(Is) is able to detect all the birds (undisguised by hills) at the horizon level. A bird as big as a stork flying $100 \mathrm{~m}$ higher than the radar location reflects an echo strong enough to be detected by the radar at the distance of $90 \mathrm{~km}$. In the daytime, at the distance of up to $60 \mathrm{~km}$ from the radar, under conditions of the open horizon, the station detects 56-83\% of migrating birds flying at the altitude of over $100 \mathrm{~m}$ above the radar location. At night, at the distance of up to $30 \mathrm{~km}$ from the radar, the station detects $74-89 \%$ of migrating birds flying at $150 \mathrm{~m}$ above the radar location. At the distances of 20 and $50 \mathrm{~km}$, the resolution capacity of the station (i.e. detecting separated echoes of two or more birds flying at a distance from each other) are $\Delta r=200 \mathrm{~m}$ and $\Delta r=400 \mathrm{~m}$, respectively; regarding the height and the tangential component; distance-wise, the capacity is $150 \mathrm{~m}$ regardless of the distance to the target.

Systematic observations of seasonal bird migration in the daytime and at night conducted during two autumn seasons and two spring seasons showed that the number of birds flying via Israel is significantly lower in spring than in autumn.
\end{abstract}

L. Dinevich, Y. Leshem, Tel-Aviv University, Ramat Aviv, Tel Aviv, Israel, Fax: 972-3-640-60-10, E-mail: dinevich@013.net

Key words: bird migration, birds, radar ornithology, radars

\section{INTRODUCTION}

MRL-5(Is) is a radar ornithological station developed in Israel on the basis of MRL-5 meteorological radar. The system performs computer-controlled bird moni- 
toring (24 hours per 7 days a week) and enables to obtain, at the interval of 15-20 minutes, updated information on bird flights over large areas within the hemispherical coverage regardless of weather conditions. MRL-5 is able to reliably detect echoes of birds as big as storks at the distance of $90 \mathrm{~km}$. The computer-controlled system is able to select bird echoes and plot ornithological charts for distances up to $60 \mathrm{~km}$. This restriction in the distance is accounted for: (a) computation rates, (b) large corpora of data to undergo sophisticated and complex analyses and (c) restricted time intervals set by aviation services for the ornithological charts to be regularly updated.

The technical parameters of the station, its problem-solving algorithms and results obtained in the long-term bird monitoring over central Israel are presented in a number of studies (Dinevich et al. 2000, 2001, 2003, 2004, 2005; Leshem et al. 2003; Dinevich and Leshem 2007, 2008, 2010; Dinevich 2009).

The aim of the present paper is to assess the accuracy of the results obtained with MRL-5(Is) and to suggest potential further development of the station. The data were collected, according to our customer's request, in order to provide information on bird migration that is of significant importance for ornithologists and ecologists, as well as for various aviation services. The observations were performed in autumn and in spring, in the daytime (from 8.00 to 17.00 ) in 2009 and at night-time (from 18.00 to $6.00)$ in 2010. The analysis of these observations alongside with the assessment of the MRL-5(Is) station accuracy are presented below.

\section{RADAR EQUATION FOR A BIRD FLOCK}

A big bird flock is a dispersed multiple radar target whose characteristics mostly resemble those of clouds and precipitation. MRL-5 has a narrow pencil-like beam and is capable of detecting at high accuracy both the location and the flight speed of a multiple (dispersed) or single radar target (Abshaev et al. 1980), including birds (Dinevich and Leshem 2008).

While deriving radar equations, we assume that the antenna beam is of symmetrical cone-like shape.

The beam width for an antenna $\theta$ (some symbols and terms are explained in Appendix) is determined by the angular width of the cone at the level of half-power of the probing signal. For a single bird or a small bird flock totally radar-covered within the scanning reflecting zone (see below) the well-known radar equation is valid:

$$
P_{r}=\frac{P_{t} G^{2} \lambda^{2} \sigma}{(4 \pi)^{3} r^{4}} k
$$

where:

$P_{r}$ - the power at the receiver entry,

$P_{t}$ - the transmitter power,

$G$ - the antenna power gain,

$\lambda$ - the wave length,

$\sigma$ - the scattering cross section (SCS) of the target,

$k$ - the total attenuation of the waveguide transmission line.

The attenuation within clouds and precipitation is not taken into account since birds are unlikely to fly under conditions of heavy rain or clouds. 
Substituting the known values for dependencies of the antenna power gain:

$$
G=\frac{4 \pi S_{e}}{\lambda^{2}}
$$

on its aperture (the actually used antenna square $S_{a}$ ) and the dependencies of the antenna power gain of its beam-width:

$$
G=\frac{4 \pi}{\theta^{2}}
$$

we can write several equivalent expressions for the radar equation.

The SCS of a single bird of dimensions big enough to be comparable with the wave length is approximately equal to its cross section area as seen at the point of the radar (plumage not taken into account). A bird's SCS depends on the flight stage and the flight course relative to the radar (Chernikov 1979, Ganja et al. 1991). These relations can be used for the purposes of bird species determination. The values of SCS for various bird and insect species measured at various wave lengths (i.e. 3.2, 5.0, 10.0 and $71.5 \mathrm{~cm}$ ) by different authors (Houghton 1964, Glover and Hardy 1966, Hajovsky et al. 1966, Konrad and Hicks 1966, Rinehart 1966, Chernikov 1979, Richardson and West 2005) as well as by the authors of this paper show quite good agreement among the data. At wave lengths of 3 and $10 \mathrm{~cm}$ bird SCS values increase depending on the species dimensions. For insects there is an inverse dependence on the wave length (compare Figure 1 in Dinevich and Leshem 2010). To calculate $\sigma$, we used the formulas suggested by Stepanenko (1973):

$$
\begin{aligned}
& \text { for } \lambda=3.2 \mathrm{~cm}, \sigma_{c^{2}}=0.6 * 10^{-24} 10^{0.1 n} R^{4} \text {, } \\
& \text { for } \lambda=10 \mathrm{~cm}, \sigma_{\mathrm{cm}^{2}}=0.28 * 10^{-25} 10^{0.1 n} R^{4} \text {, }
\end{aligned}
$$

where:

$n$ - the radar reflectivity of the experimental target $(\mathrm{dB})$

$R$ - the distance to the target (m).

The coefficients in the formulas are calculated based on the constant parameters of the antenna, the receivers and the transmitters. The principle for bird echo isolation used in this study is described in Dinevich et al. (2001). The SCS values on the second channel are higher than those on the first channel, thus meaning that our findings are close to the results obtained by other authors (Chernikov 1979).

In some cases we classified the echoes with the corresponding values to insect echoes. Their $S C S$ values at $3 \mathrm{~cm}$ wave length were higher than those at the $10 \mathrm{~cm}$ wave length. In these cases visual observations showed large presence of insects near the ground (midges, moths, mosquitoes, etc.) well seen in the light of street lamps, car headlights and even in darkness at short distances. According to radiosonde data, the ground air in these cases was characterized by higher humidity, with a weak wind directed to west-northwest.

For example, in a case study on 25 October 2002 at 21.30 the first inversion level was determined at the height of $800-1200 \mathrm{~m}$. The moon was seen through sparse light. At those altitudes, at both MRL-5 channels one could see a weak but broad echo from an invisible atmospheric inhomogeneity about $500 \mathrm{~m}$ thick. This may suggest a possible presence of vertical air flows in the sub-inversion air level, that triggered 
accumulation of various admixtures including insects. A specific feature of this echo was the difference in its differential reflectivity values measured at its upper $v s$ bottom level. The differential reflectivity was calculated according to the following equation:

$$
d P=\frac{P_{\|}}{P_{\perp}},
$$

where:

$d P \quad$ - the differential reflectivity (a dimensionless quantity),

$P_{\| \mid}$- the power of the reflected signal in $\mathrm{mW}$ (a horizontal polarization wave was both received and radiated)

$P_{\perp} \quad$ - the power of the reflected signal in $\mathrm{mW}$ (a vertical polarization wave was both received and radiated).

The polarization was altered pulse-by-pulse. At the repetition rate of 500 pulses per second the polarization was modified every $1 / 500 \mathrm{~s}$. We assume that during such a short period of time the targets we studied (clouds, atmospheric inhomogeneities and birds) could not change their status. The signal was averaged over approximately 250 pulses. The values of the differential reflectivity at the upper part of the echoes was close to unity (or $0 \mathrm{~dB}$ ), which is typical of the reflection from cloud particles or of boundaries of atmospheric layers with high temperature-humidity gradient (Shupyatsky 1959, Chernikov and Shupyatsky 1967, Zrnic and Ryzhkov 1998). This can be accounted for by the isotropic reflection properties of atmospheric inhomogeneities (visually unobservable thermics or mesofronts), small-size spherical cloud drops that are chaotically oriented in the medium of small crystals (however, in our case, there could not have been any such crystals).

The differential reflectivity from the bottom part of the echo was $>1$, which is typical of non-spherical reflectors horizontally oriented in the space (Dinevich et al. 1994). Thus it suggests that the bottom part of the echo was formed not only due to the temperature-humidity gradient, but also due to the presence of horizontally oriented dipoles present within the sub-inversion layer - these dipoles can be only insects or oblong plant seeds. In addition, it should be emphasized that the echoes in all the cases shifted in the direction and at the speed of the wind at this level (according to the reading of the radio-probing closest in time). It allows us to conclude that that the echoes we observed on both channels were insect echoes. The SCS value was always higher at the $3 \mathrm{~cm}$ wave length than on the $10 \mathrm{~cm}$ band, which is in accordance with conclusions drawn by other researchers (Glover and Hardy 1966, Chernikov 1979).

Our results show that the $10 \mathrm{~cm}$ channel is more efficient in detecting birds, yielding better results in both the number of detected birds and the detection distance. This can be due to the fact that the $10 \mathrm{~cm}$ channel has a higher potential and a wider beam "catching" birds over a larger area. For this reason, we selected the second channel $(\lambda=10 \mathrm{~cm})$ as better for bird monitoring. For the purposes of this paper, it is enough to use the averaged data. Table 1 presents the mean SCS for various bird species at the radar wave length $\lambda=10 \mathrm{~cm}$ or less.

While deriving the radar equation for a big bird flock we followed the approach traditionally used for a dispersed multiple target such as clouds and precipitation. This approach is related to the concept of reflecting area (Abshaev et al. 1984). With 
the reflecting signal being at a certain delay from the moment of probing and the delay being expressed as:

$$
t=\frac{2 r}{c}
$$

where:

$r$ - the distance to the target,

$c$ - the light speed,

the volume of the reflectivity area is expressed as:

$$
V(t)=(r \theta)^{2} \frac{c \tau}{2}
$$

where:

$\tau$ - the duration of the probing pulse.

With the radar beam being symmetrical, this volume has a cylinder-like shape having at its base a circle of radius:

$$
R=r \theta
$$

and the height of:

$$
h=\frac{c \tau}{2} \text {. }
$$

Table 1

Scattering cross-section (SCS) values for various bird species according to calculations

\begin{tabular}{|l|c|c|c|}
\hline \multicolumn{1}{|c|}{ Bird species } & Body length $(\mathrm{cm})$ & Body width $(\mathrm{cm})$ & SCS $\left(\mathrm{cm}^{2}\right)$ \\
\hline Sparrow & 5 & 3 & 15 \\
\hline Pigeon & 8 & 4 & 30 \\
\hline Starling & 6 & 3 & 15 \\
\hline Lark & 10 & 6 & 60 \\
\hline Gull & 15 & 8 & 120 \\
\hline Albatross & 30 & 12 & 400 \\
\hline
\end{tabular}

The axis of the cylinder is angled relative to the Earth surface, with the angle being equal to the tilt angle $\gamma$ of the antenna.

A typical structure of a cloud is significantly different from that of a bird flock. The typical thickness of a cloud is less than its horizontal dimension and is less than the diameter of the reflective area at smaller distances. Unlike clouds, birds usually fly in the daytime approximately at the same altitude, thus a bird flock forms a relatively thin horizontal layer. For this reason, the classic radar equation valid for clouds and precipitations (Abshaev et al. 1984) is not applicable for a big bird flock flying within a small altitude range.

The square of the area covered by a bird flock flying at the same altitude and getting entirely within the reflectivity area depends on the tilt angle $\gamma$. At small distances it is calculated as:

$$
S=\frac{(r \theta)^{2}}{\sin \gamma}
$$


while at large distances as:

$$
S=\frac{r \theta c \tau}{2 \cos \gamma}
$$

The critical angle at which one should shift from the equation (1) to (11) or (12) is:

$$
\gamma_{\kappa}=\operatorname{arctg} \frac{2 r \theta}{c \tau} \text {. }
$$

In case $\gamma>\gamma_{k}$, formula (11) is valid, otherwise (12) should be applied.

As a rule, the tilt angle that allows to detect birds is less than the critical angle and close to zero: $\gamma_{k}>\gamma \approx 0$, hence we can express the square of the layer as:

$$
S=\frac{r \theta c \tau}{2} .
$$

Hereafter, we are going to use the expression (1) for the square of the layer.

When a single bird is flying at the average distance $l$ from a next flying bird and occupies the fraction of the square equal to:

$$
S_{b}=\frac{\pi l^{2}}{4}
$$

the number of birds simultaneously exposed to the radar radiation is:

$$
n=\frac{S}{S_{b}} \text {. }
$$

Assuming that the signal from a bird flock is scattered incoherently, at the SCS value for a single bird $\sigma_{b}$ we obtain the SCS for the volume surrounding the bird as:

$$
\sigma=\frac{S}{S_{b}} \sigma_{b}
$$

Substituting this value into the formula (1) and using (14) for $S$, we derive the radar equation for a bird flock flying at a large distance from the radar at the tilt angle close to zero:

$$
P_{r}=\frac{P_{t} G^{2} \lambda^{2} \theta c \tau \sigma_{b}}{2(4 \pi)^{3} r^{3} S_{b}} k=\frac{P_{t} G^{\frac{3}{2}} \lambda^{2} c \tau \sigma_{b}}{2(4 \pi)^{2.5} r^{3} S_{b}} k
$$

where the dependency:

$$
\theta=\sqrt{\frac{4 \pi}{G}}
$$

is used in the right-hand equation.

For a bird flock seen at a significantly high tilt angle that is still less than the critical angle, the radar equation is as follows:

$$
P_{r}=\frac{P_{t} G^{2} \lambda^{2} \theta c \tau \sigma_{b}}{2(4 \pi)^{3} r^{3} S_{b} \cos \gamma} k=\frac{P_{t} G^{\frac{3}{2}} \lambda^{2} c \tau \sigma_{b}}{2(4 \pi)^{25} r^{3} S_{b} \cos \gamma} k .
$$


Therefore, the power of the signal reflected from a bird flock is inversely proportional to the cube of the distance.

It should be noted that at night birds usually fly within a larger altitude range and at higher density within the radar scan. In this case the power of the signal reflected from a bird flock covering a certain volume is similar to the power of signal reflected from a cloud and thus is inversely proportional to $r^{4}$

As an example, we calculated the power of signal received on the first MRL-5 channel, reflected from a flock of sparrows $\left(\sigma_{b}=1.5 \times 10^{-3} \mathrm{~m}^{2}\right)$ flying at an average distance of $30 \mathrm{~m}$ from each other $\left(S_{b}=700 \mathrm{~m}^{2}\right)$ at the height of $500 \mathrm{~m}$ and the distance from the radar of $30 \mathrm{~km}$. The tilt angle at which the target is viewed is $\gamma=\arcsin 0.05$ $=2.9^{\circ}, \cos \gamma \approx 1$ and the critical angle is:

$$
\gamma_{k}=\operatorname{arctg} \frac{2 \times 10000 \times 0.009}{3 \times 10^{8} \times 10^{-6}}=31^{\circ} .
$$

Therefore, one can apply the radar equation from (18). Substituting the parameters of the MRL-5 first channel: $\lambda=3.2 \mathrm{~cm}, \theta=0.5^{\circ}=0.009 \mathrm{rad}, G=1.2 \times 10^{5}, \tau=1 \mu \mathrm{s}$, $P_{t}=2.5 \times 10^{5} \mathrm{~W}$, and $k=0.3(-5 \mathrm{~dB})$ into $(18)$, we obtain $P_{r}=7 \times 10^{-11} \mathrm{~W}$, which is a value by three orders of magnitude higher than $2.5 \times 10^{-14} \mathrm{~cm}(-136 \mathrm{~dB} / \mathrm{W})$, which is the threshold sensitivity of the receiver. Therefore, the power of a received signal is not a factor restricting the distance of bird radar monitoring.

Using the radar equation for birds, we can, on the basis of a received signal, evaluate the complex parameter of specific $S C S$, i.e. the $S C S$ per a square unit $\sigma_{b} / S_{b}$ of the bird-covered layer. If, in addition to the $\sigma_{b} / S_{b}$ value, we have some data on the averaged distance between the next-flying birds, we can calculate the SCS for a single bird. And vice versa - if we have additional data on the SCS for a single bird, we can evaluate the distance between next-flying birds and thus evaluate the number of birds in the flock. The number of birds in a flock is an important parameter per se; the SCS of a single bird enables to evaluate its size and thus determine its species with a certain probability. However, none of these parameters can be evaluated solely on the basis of the amplitude of a signal yielded by a single probing.

When taking into account the specific geometric features characterizing flight patterns of various bird species (Shestakova 1971), as well as various parameters of radar echo (fluctuation, polarization, two-wave mode, Doppler, spatial and temporal characteristics) we can evaluate several important ornithological parameters (Zavirukha et al. 1977, Ganja et al. 1991, Dinevich et al. 1994, Zrnic and Ryzhkov 1998, Leshem et al. 2003).

For example, the analysis of fluctuation parameters of radar echo enables isolating signals from single birds at high accuracy (Dinevich et al. 2004). Sound tracking of the fluctuations provides data on the changes in spatial orientation of the bird and the pattern of its wings' flapping. Based on the radar equation formulated above, the SCS of a bird can be measured, thus providing data on the bird's size. These parameters alongside with the pattern of the bird's movement in the space enable to determine its species (Shestakova 1971; Doviak and Zrnic 1984; Dinevich and Leshem 2007, 2008). By putting together the measurements of the radar reflectivity within each radar scan as well as the data on bird species and SCS, one can approximately 
assess the number of birds in a flock and their concentration within a certain space. However, a detailed analysis of this issue is beyond the scope of the present paper.

\section{RESTRICTIONS ON THE BIRD ECHO MONITORING DUE TO THE CURVATURE OF THE EARTH}

According to Abshaev et al. (1984), the maximum range of vision $r_{\max }(\mathrm{km})$, determined by the curvature of the Earth, depends on the height of the radar $h_{1}$ and the height of the target $h_{2}$, thus:

$$
r_{\max }=4.18\left(\sqrt{h_{1}}+\sqrt{h_{2}}\right) .
$$

In most cases, the height of the radar is significantly lower than that of the target, consequently the first summand in the equation (21) is negligible:

$$
r_{\max }=4.18 \sqrt{h_{2}} \text {. }
$$

In the inverse formulation, this formula determines the minimum height (in metres) at which a target is visible at a certain distance (in kilometres):

$$
h_{\min }=5.7 \times 10^{-8} r^{2} \text {. }
$$

While determining the maximum range of vision on the basis of equations (21), (22) and (23), the beam width is assumed to be small and it is supposed that the radar beam can be at zero angle relative to the Earth surface. However, at extremely small tilt angles the beam "gears" the Earth surface. This leads to a change in the amplitude of the received signal, and what is more significant, to emergence of ground clutter reflections (those from hills, woods, buildings and other installations). With the bird signal being relatively weak, those ground clutter reflections disguise it, making it indistinguishable.

Even at large tilt angles, the target signal may be disguised by the ground clutter reflections due to the side lobes of the beam. Without taking the side lobes into account, we would assume that all the radiation is concentrated on the main beam lobe. Then in case the tilt angle exceeds half of the beam width, i.e. $\gamma>\theta / 2$, the entire beam is located above the Earth surface enabling us to get rid of any reflections from lower ground clutter.

It should be noted that at large distances there are no ground clutter reflections since the radar beam moves away from the Earth surface due to its curvature. At the lower position of the antenna, when $\gamma=\theta / 2$, the minimum height of a target depends on the distance to it

$$
h_{\min }>\frac{r \theta}{2}
$$

Therefore, at small distances the main factor enabling radar monitoring of flying birds is a tilt angle value that exceeds a certain criterion threshold $r \theta / 2$. At larger distances the tilt angle can be decreased, although in this case, the curvature of the Earth will have an impact. Both factors can be properly taken into account using a formula that yields the value of the minimum height (in metres) depending on the 
distance (in kilometres), this values being the root of sum-of-squares of the equations (23) and (24):

$$
h_{\min }>\sqrt{\left(\frac{r \theta}{2}\right)^{2}+3.25 \times 10^{-15} r^{4}} .
$$

In (25) the first summand under the square root sign depends on the beam width, while the second summand does not. As the beam width decreases, the radar ability for detecting low-flying birds increases; the bottom limit of the height cannot be equal to zero, as it depends on the curvature of the Earth. Abshaev (1980) suggests a different formula for calculating the minimum height at which a target can be detected at normal refraction values:

$$
h=r \sin \theta+\beta r^{2},
$$

where:

$r$ - the distance to the target,

$\theta$ - the tilt angle,

$\beta=6 \times 10^{-5} \mathrm{~km}$.

The second summand takes into account the difference between the radius of the Earth curvature and the beam trajectory under conditions of normal refraction. At normal refraction values, if the radar is located at sea level and the beam is $0.5^{\circ}$, the minimum height at which birds can be detected is $400 \mathrm{~m}$ at the distance of $50 \mathrm{~km}$, and $700 \mathrm{~m}$ at the distance of $100 \mathrm{~km}$. When the radar is located above sea level, these values are significantly lower. For instance, MRL-5 radar in Latrun (Israel) is located at $270 \mathrm{~m}$ above sea level. At normal refraction conditions, this radar is able to detect all the birds at the distance of $25 \mathrm{~km}$ at the horizon level $\left(\theta=0^{\circ}\right)$ even at a certain negative tilt angle. At distances of $50 \mathrm{~km}$ and $100 \mathrm{~km}$, this radar can monitor birds flying at the altitudes of $100 \mathrm{~m}$ and $400 \mathrm{~m}$ relative to the radar location level, respectively.

These calculations show that at lower altitudes the main factor restricting the range of radar vision is not the radar station potential, but rather the curvature of the Earth, the height of the radar above sea level, openness of the horizon and the beam width. This means that the actual minimum height at which birds can be detected depends on the parameters of the underlying ground, in particular on its hilliness and the altitudes at which birds are flying. Table 2 presents the maximum values for ranges of visions accessible by MRL-5 when birds are flying at the minimum height. In fact, these distances are different for different scanning directions and depend on the extent of "openness" of a particular direction for the radar beam.

Table 2

Distances at which birds can be detected depending on their flight altitude, experimentally established by means of MRL-5 in Latrun (at zero tilt angle)

\begin{tabular}{|c|c|c|c|c|c|}
\hline Flight altitude & $200 \mathrm{~m}$ & $250 \mathrm{~m}$ & $300 \mathrm{~m}$ & $400 \mathrm{~m}$ & $600 \mathrm{~m}$ \\
\hline Detection distance & $20-25 \mathrm{~km}$ & $25-30 \mathrm{~km}$ & $30-40 \mathrm{~km}$ & $40-50 \mathrm{~km}$ & $50 \mathrm{~km}$ \\
\hline
\end{tabular}




\section{ACCURACY AND RESOLUTION CAPACITY OF RADAR BIRD MONITORING}

Accuracy and resolution capacity of the radar while detecting a single bird or a bird flock is determined by well-known dependencies characterizing radar location of a dispersed multiple target (Battan 1959). A highly important component of our computer-controlled station is the system of averaging over space and time, which enables to isolate the target signal against the background noise. However, averaging over space and time leads to a certain expansion of the signal in comparison to the radar's own signal. For this reason, one should take into account the parameters of the entire station rather than the radar itself. The algorithm and the design of the system for averaging over space and time is described in detail elsewhere (Battan 1959); it is important to point out that its main parameters are: (a) the number of averaged probing pulses $n_{1}$ while averaging over distances and (b) the number of averaged scanning intervals $n_{2}$ while averaging over the angle. For the system to perform efficiently, the product of these coefficients has to obey the condition $n_{1} \times n_{2}>50$. If the amplification band of the radar receiver matches the frequency band of the probing signal, the accuracy and resolution capacity of the radar in bird monitoring can be calculated by the following formula:

$$
\Delta r=c \tau \sqrt{1+\frac{n_{1}^{2}}{2}} .
$$

In the tangential direction, the accuracy and resolution capacity obey the expression:

$$
\Delta r=r \sqrt{\frac{1}{2} \theta^{2}+n_{2}^{2} \omega^{2} T^{2}}
$$

where:

$\omega$ - the angle velocity of antenna rotation,

$T$ - the scanning interval.

At small tilts, the magnitude of the spike is chosen to be equal to the beam width, so:

$$
\Delta r \approx 1.2 r \theta
$$

Height-wise, the accuracy and resolution capacity are calculated as:

$$
\Delta r=r \sqrt{\frac{1}{2} \theta^{2}+\theta_{s}^{2}},
$$

where:

$\theta_{s}-$ the value of a spike at the tilt angle.

We can now calculate the accuracy and the resolution capacity over the coordinates for the computer-controlled station based on MRL-5 meteo-radar. The parameters of the radar are as follows: pulse duration $\tau=1 \mu \mathrm{s}, n_{1}=4, n_{2}=18, \theta=0.5^{\circ}, p=$ $0.017 \mathrm{rad}, \omega=36 \% \mathrm{~s}=0.314 \mathrm{rad} / \mathrm{s}, T=2 \mathrm{~ms}$. The power of bird echo does not usually exceed $30 \mathrm{dBZ}$, which means that the beam usually catches the echo within its centre. 
Therefore, one can reliably assume that the width of the active segment of the beam able to detect a weak signal reflected from a bird does not exceed $\theta / 2=0.25^{\circ}=$ $0.0085 \mathrm{rad}$. Thus for distances of $20 \mathrm{~km}$ and $50 \mathrm{~km}$ the resolution capacity required for separating bird echoes is, with regard to the height and the tangential component, $\Delta r=200 \mathrm{~m}$ and $\Delta r=400 \mathrm{~m}$, respectively. The resolution capacity distance-wise does not depend on the distances and is $150 \mathrm{~m}$.

\section{THE EXPERIMENTALLY ESTABLISHED PROBABILITY OF BIRD DETECTION AT VARIOUS DISTANCES}

The method for assessing the probability of bird detection at various distances from MRL-5(Is) is described elsewhere (Dinevich and Leshem 2008). Table 3 shows some findings from the study relevant for a certain scanned area (Latrun in Israel, radar location at about $300 \mathrm{~m}$ a.s.l.). In addition to Table 3, we present below two ornithological charts of bird migration at night and in the daytime (Fig. 1 and 2).

Figure 1 shows a chart of the bird flow on 20 October 2010 at 19.24, within the radius of $40 \mathrm{~km}$ from the radar. In the main part of the figure we see multiple echoes from small bird flocks and single birds. This migration pattern is typical of night-

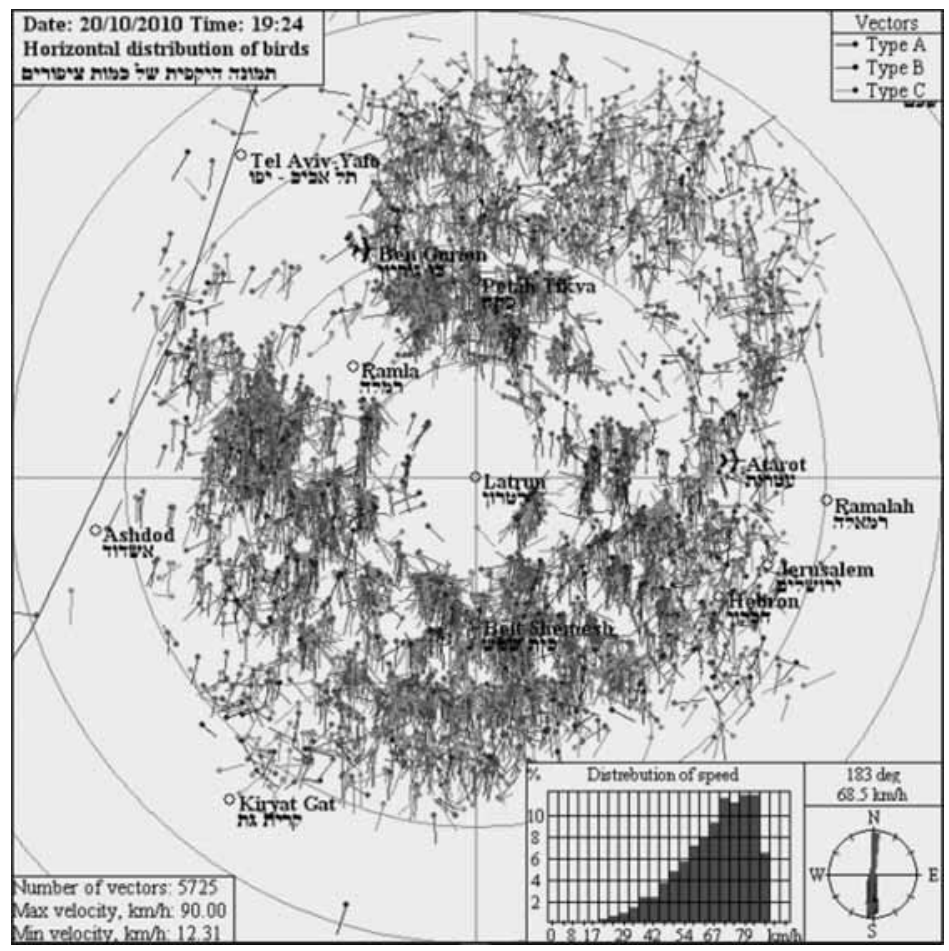

Fig. 1. Chart of the bird flow on 20 October 2010, at 19.24 (night-time). Scan radius $-40 \mathrm{~km}$, number of vectors -5725 , maximum flight speed $-90 \mathrm{~km} / \mathrm{h}$, minimum speed - ca 12 $\mathrm{km} / \mathrm{h}$. The right bottom part shows flight speed distribution. The arrow within the circle indicates the average flight direction for the total of the birds. 


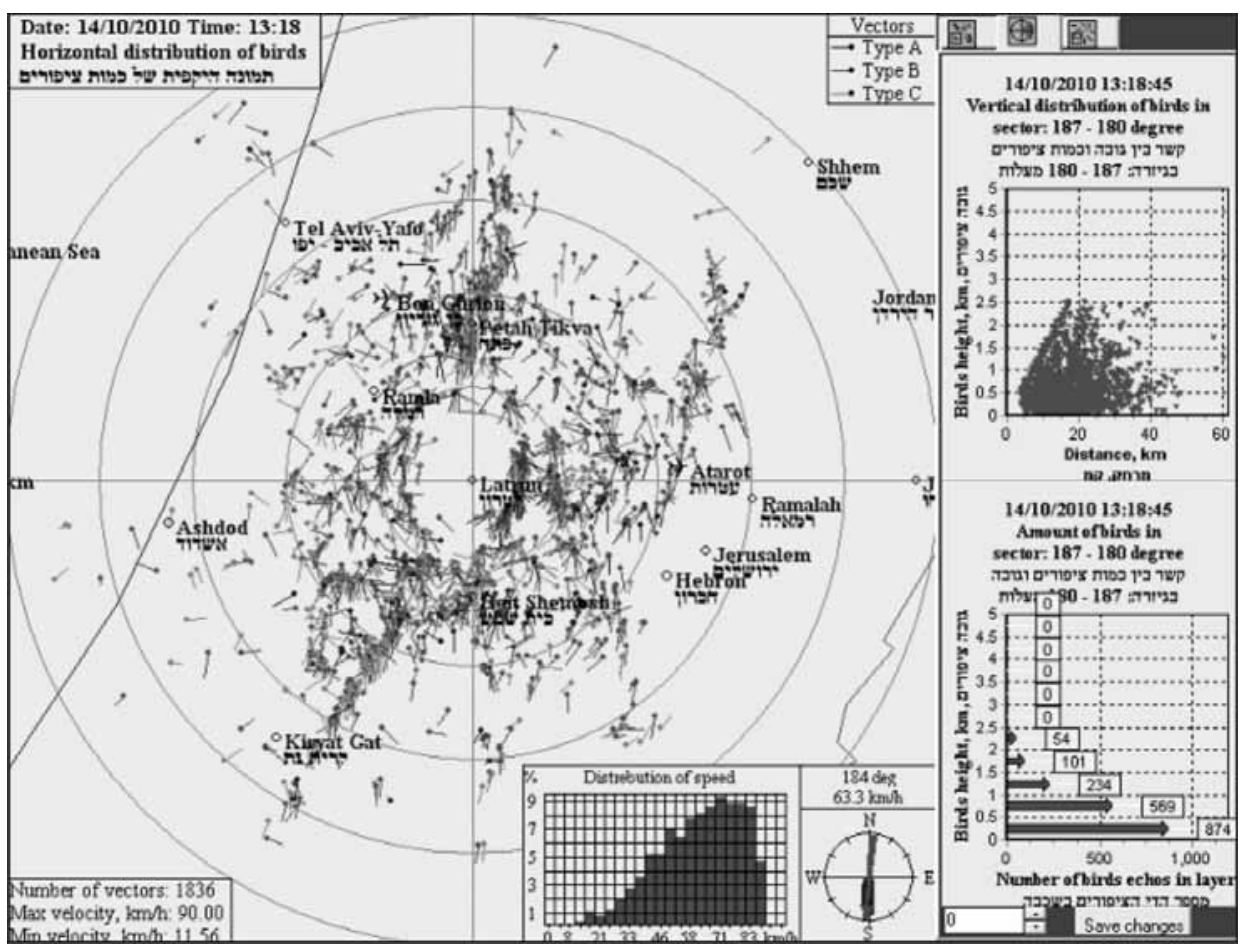

Fig. 2. Chart of the bird flow on 14 October 2010, at 13.18 (daytime). Scan radius $-50 \mathrm{~km}$, number of vectors - 1836, maximum flight speed - $90 \mathrm{~km} / \mathrm{h}$, minimum speed - ca 12 $\mathrm{km} / \mathrm{h}$. The right bottom part shows flight speed distribution. The arrow within the circle indicates the average flight direction for the total of the birds. The right panel shows the projection of all the echoes within the scan area onto the vertical plane and (below) the distribution of the vectors over the altitude (by 500 m layers).

time. One can see 5725 vectors oriented from the north to the south, almost all of them originally coloured red. Those are vectors of echoes from birds whose flight speed is variable, and flight direction deviates from a straight line by not more than $20 \%$. At the colourful original there are a few blue-coloured vectors of echoes from birds flying strictly along a straight line at a stable speed. Vectors of echoes from birds flying at highly variable speed and in frequently changing directions would be originally marked brown. The limitations are mostly accounted for by the flight altitudies and the orography of the surface (it is covered by hills at low altitudes).

\section{Table 3}

The probability $(p)$ of bird detection at various distances at night and in the daytime

$$
(\lambda=10 \mathrm{~cm})
$$

\begin{tabular}{|l|c|l|l|l|l|c|}
\hline \multirow{2}{*}{} & \multicolumn{7}{|c|}{ Distance from the radar (km) } \\
\cline { 2 - 7 } & $5-10$ & $10-20$ & $20-30$ & $30-40$ & $40-50$ & $50-60$ \\
\hline$p$ at night (\%) & 100 & $86-100$ & $74-89$ & $35-52$ & $14-41$ & $\sim 20$ \\
\hline$p$ in the daytime (\%) & 100 & $93-100$ & $77-89$ & $68-86$ & $61-82$ & $56-83$ \\
\hline
\end{tabular}


Figure 2 presents a chart of the bird flow on 14 October 2010 at 13.18 . In the middle part of the chart we can see long strips of bird flock echoes (some as long as $90 \mathrm{~km}$, sometimes interrupted with gaps), as well as multiple echoes from small flocks and single birds. Flight pattern of this type is typical of the conditions of a developing convection. Unlike night flights, daytime flights of migrating birds are most often shaped as strips oriented in parallel with the coast line.

Graphs of the mean altitudes of maximum bird concentrations are presented in Figure 3. At the altitudes within zone below $500 \mathrm{~m}$, the distance at which birds can be detected, both in the daytime and at night, is below $30 \mathrm{~km}$. The higher birds fly, the larger is the distance at which they can be detected. In spring the altitudes of maximum bird concentrations, both during the day and at night, increase month by month. In autumn, both during the day and at night, the altitudes of maximum bird concentrations vary from day to day, but do not have a distinct pattern for a specific month. The average altitude of migration is significantly higher in spring than in autumn: 964 vs $601 \mathrm{~m}$ in 2009 and 834 vs $642 \mathrm{~m}$ in 2010 ( $t$-test: $p<0.001$ in both cases).

As it was mentioned above, the analysis of the charts and the graphs shows that, when the parameters of the terrain and flight altitudes are taken into account, not less than $35-52 \%$ of night-flying birds can be detected at the distance of $40 \mathrm{~km}$. At the distance of up to $60 \mathrm{~km}$, MRL-5(Is) is able to detect about $20 \%$ of night-flying birds. However, this is true only if the flight altitude is above $400 \mathrm{~m}$. The daytime-flying bird
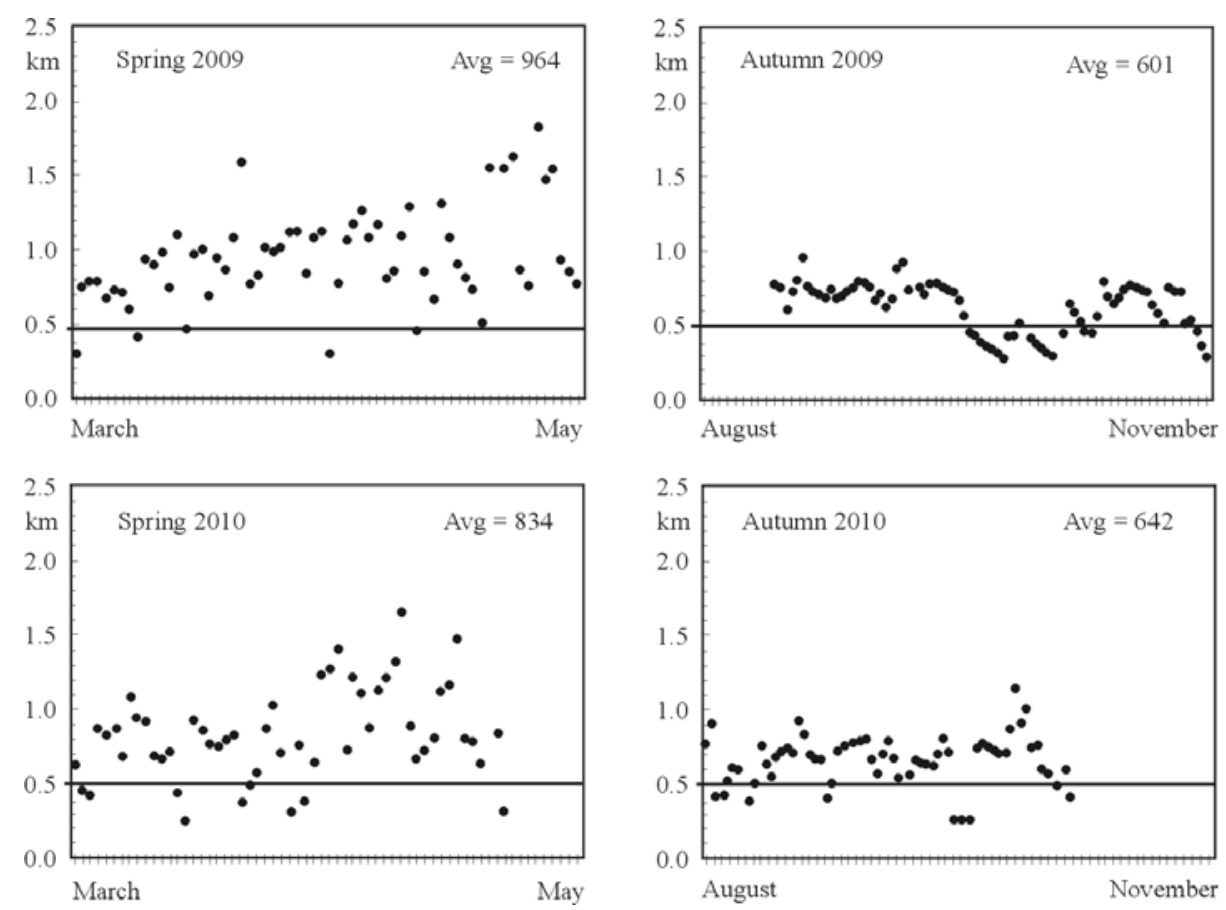

Fig. 3. The daily mean altitudes of maximum bird concentrations during spring and autumn. $A v g$ - average altitudes of flights for the whole periods. The charts for 2009 concern daytime monitoring, for 2010 - night-time. 
species (e.g. storks, pelicans, eagles, buzzards, etc.) are usually bigger than night migrants. The SCS of these species and, consequently, their echoes are larger than those of night-flying birds. While flying, these larger birds often take advantage of convective atmospheric flows that form due to inhomogeneity of the ground surface, as well as to the upland-valley breeze processes. These birds often form strips as long as 90-100 km. The analysis of our daytime observations (Table 3) shows that MRL-5(Is) is able to detect not less than $80 \%$ of migrating birds at the distance of up to $30 \mathrm{~km}$, which is close to the number of birds detected at night. As to the daytime-flying birds, MRL-5(Is) is able to detect up to $60 \%$ of them at the distances of up to $60 \mathrm{~km}$. The detection distance and the probability of achieving a larger detection rate correlates well with the flight altitude and the degree to which the ground surface the birds fly over is open for the radar beam. In favourable conditions, MRL-5(Is) is able to detect up to $83 \%$ of all flying birds within the maximum scan radius.

It is clear that there are significant variations in the system ability to detect birds within the scan area of $60 \mathrm{~km}$ radius. The analysis described above enables to determine several factors that may account for these differences:

1. In some cases, birds fly beneath the radar beam level.

2. A part of birds crossing central Israel fly over the Jordan Valley, which is situated much below the level accessible by means of MRL-5(Is). The lower part of the Jordan Valley near the Dead Sea is located $500 \mathrm{~m}$ below sea level, i.e. about $800 \mathrm{~m}$ below the radar location.

3. The character of the terrain lying beneath the route of migrating birds is an important factor, since those routes often go along valleys located between high hills and thus unreachable for the radar.

4. The completeness of the data depends on the systematic observations, including uninterrupted schedule and interpolation for periods between scans.

\section{NUMBERS OF BIRDS FLYING OVER CENTRAL ISRAEL IN AUTUMN AND SPRING}

In view of considerations presented above, the quantitative assessment of absolute numbers of birds flying over Israel should be regarded as approximate only. However, taking into account the vast corpus of seasonal data, we believe that relative characteristics (i.e. data comparison over extended periods of time) can be representative for various assessments.

Figure 4 presents the numbers of birds that flew over Central Israel in spring and autumn migration periods, while Figure 5 illustrates the seasonal pattern of migration by days in 2009 and 2010. The number of birds is larger in autumn than in spring both in diurnal and nocturnal migrants. For diurnal migrants in 2009 average migration density in spring was 433 birds/hour $/ \mathrm{km}^{3}$ while in autumn 582 birds $/$ hour $/ \mathrm{km}^{3}$ (1 : 1.34 ratio). In 2010 for nocturnal migrants these numbers were 419 birds/hour/ $\mathrm{km}^{3}$ in spring and 1099 birds/hour $/ \mathrm{km}^{3}$ (1:2.62). For both groups of migrants differences between seasons were highly significant ( $t$-test: $p<0.001$ in both cases). It could be supposed that bird species migrating in the daytime have lower wintering 
loses than bird species flying at night, but it needs confirmation by comparisons between subsequent migration seasons: autumn migration in one year with the spring migration next year. A long-term study and a massive database are needed in order to conclude if these suggestions are valid for regional relevance only or we may speak of a steady year-to-year pattern.
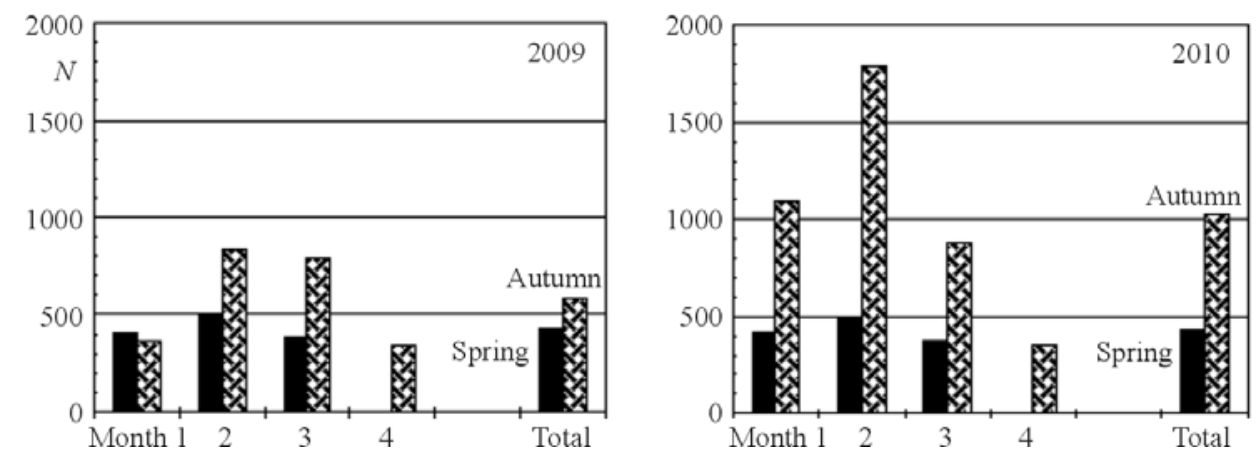

Fig. 4. Numbers of migrating birds (in birds $/$ hour $/ \mathrm{km}^{3}$ ) in subsequent months of migration and the total seasonal averages of bird numbers (spring: Month 1 - March, 2 - April, 3 - May; autumn: 1 - August, 2 - September, 3 - October, 4 - November). The chart for 2009 concerns daytime monitoring, for 2010 - night-time.
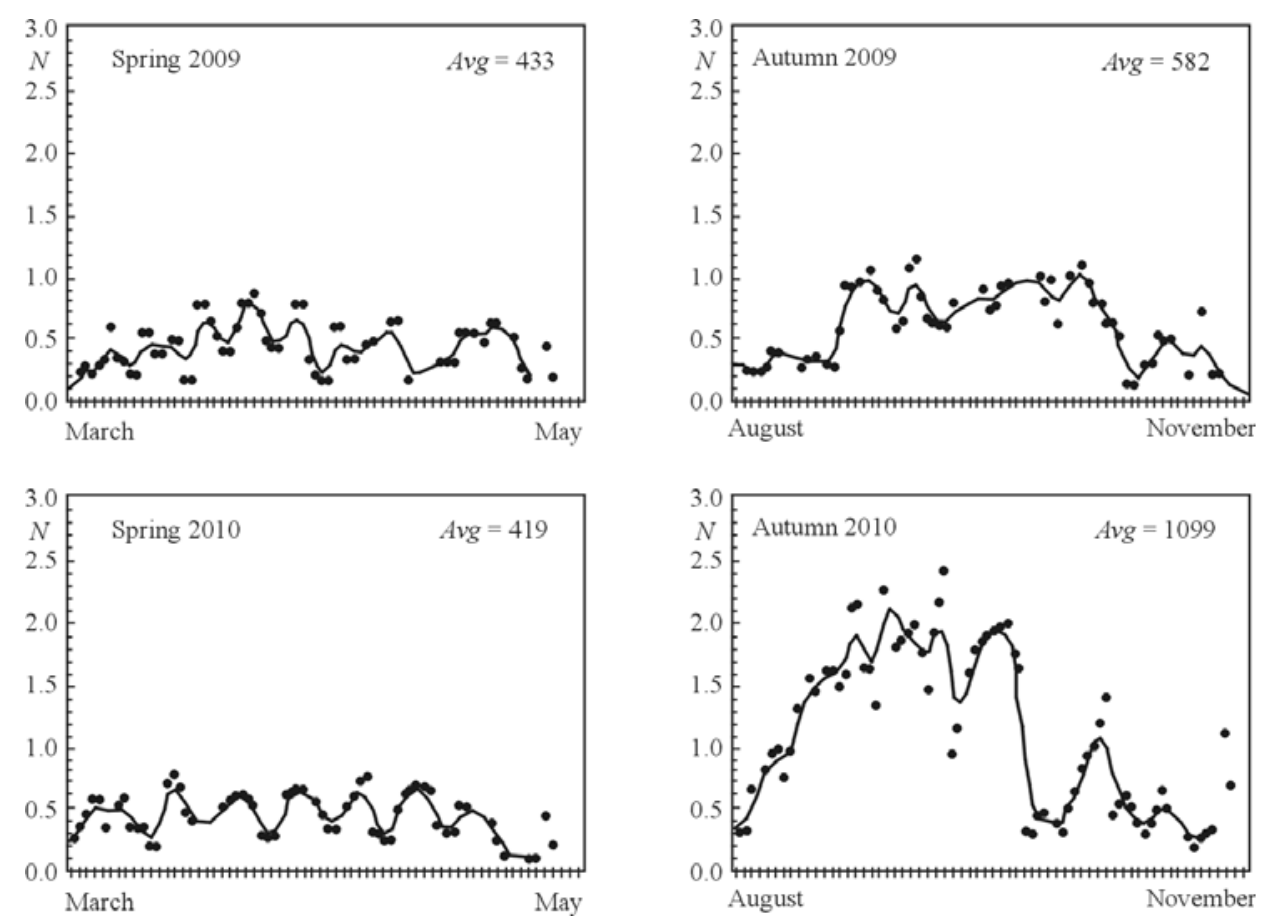

Fig. 5. The mean numbers of migrating birds per hour and cubic kilometer of air in autumn in daytime (2009) and in spring at night-time (2010). Average densities for the whole periods are given on graphs $(A v g)$. 


\section{GENERAL CONCLUSIONS}

1. Under conditions of normal refraction at distances of 5-25 km, MRL-5(Is) is able to detect all the birds (undisguised by hills) at the horizon level $\left(\theta=0^{\circ}\right)$; in some directions, the birds can be detected even at a certain negative angle.

2. At low altitudes, the main factor restricting the distance of radar bird monitoring is not the potential of MRL-5, but rather a set of other factors such as:

- the curvature of the Earth,

- the height of the radar (above sea level),

- the openness of the horizon for the radar beam

- the beam width.

A bird as big as a stork flying at over $400 \mathrm{~m}$ a.s.l. (i.e. $100 \mathrm{~m}$ higher than the radar) reflects an echo strong enough to be detected by the radar at the distance of $90 \mathrm{~km}$.

3. In the daytime, at the distance of up to $60 \mathrm{~km}$ from the radar, under conditions of the open horizon, the station detects $56-83 \%$ of migrating birds flying at the altitude of over $100 \mathrm{~m}$ higher than the radar. The undetectable birds either fly below the beam level or are disguised by hills.

4. At night, at the distance of up to $30 \mathrm{~km}$ from the radar, the station detects $74-89 \%$ of migrating birds flying at the altitude of over $250 \mathrm{~m}$ a.s.l. (i.e. even $50 \mathrm{~m}$ below the radar).

5. At distances of $20 \mathrm{~km}$ and $50 \mathrm{~km}$, the resolution capacity of the station (i.e. detecting separated echoes of two or more birds flying at a distance from each other) $\Delta r=200 \mathrm{~m}$ and $\Delta r=400 \mathrm{~m}$, respectively, regarding the height and the tangential component; distance-wise, the capacity is $150 \mathrm{~m}$ regardless of the distance to the target.

6. Systematic observations of seasonal bird migration in the daytime and at night conducted during two autumn seasons and two spring seasons showed that the number of birds flying via Israel is significantly lower in spring than in autumn. The observed difference most probably reflects the mortality of some birds wintering in Africa. Our findings give a hope to approximately assess the survival of migrants during winter and the percentage of bird population increase after the breeding season in the "home environment" in Europe. A long-term study and a massive database are essential in order to conclude if these findings are of regional relevance only or if we may speak of a steady year-to-year pattern.

\section{ON THE POTENTIAL DEVELOPMENT OF THE SYSTEM}

According to several studies, including those by the authors of the present paper, it has been shown that fluctuation parameters of radar echo can be effectively used to distinguish signals reflected from single-flying birds at high accuracy. A sound representation of the fluctuation gives a good understanding of the bird orientation in space and the wing-flapping pattern. Using the radar equation one can measure the $S C S$ of a bird and thus determine its size at a certain degree of accuracy. These 
characteristics together with the pattern of movement give some indication on the bird's species. By measuring the level of radar reflectivity within each scanning area and knowing the species and SCS of the birds, we can approximately assess their numbers and spatial concentration.

The algorithm for bird echo isolation developed for MRL-5(Is) should be adapted for Doppler radars. This will enable both to significantly reduce the time needed for data update and to increase the accuracy. The issues related to the development of the adapted algorithm and corresponding software is a matter of further research aimed at advancement of MRL-5(Is), which goes beyond the scope of this paper. We hope that planned establishing of the Center for Bird Migration Monitoring in Latrun (Israel) will open new perspectives for achieving this goal.

In view of the specific orographic characteristics of the territory of Israel, at least six radar stations of MRL-5(Is) type should be installed in order to ensure detection of at least $80 \%$ of migrating birds. Two of these stations should be used for observations over the lower parts of the Jordan Valley.

\section{ACKNOWLEDGEMENTS}

The authors express their gratitude to the Department of Science of the Ministry of Defense of the State of Israel for the financial support of the project, to the administration of Tel Aviv University for their continuous interest in the project, as well as they deep thank to several people for the important contribution: to Alexander Kapitannikov and Oleg Sikora who assisted in designing components of the software; to Vladimir Dinevich who helped to install the radar, to Valery Garanin and Dmitry Shtivelman who provided the technical maintenance of the radar, and Prof. Arcady Shupyatsky for his decades long friendship and constructive work on this paper. The authors wish also to specially acknowledge the contribution made by Prof. Lev Kaplan.

\section{REFERENCES}

Abshaev M., Burtsev I., Vaksenburg S., Shevela G. 1980. [Guide for use of the MRL-4, MRL-5 and $M R L-6$ radars in urban protection systems.] Hydrometeoizdat, Leningrad. (In Russian).

Abshaev M., Kaplan L., Kapitannikov A. 1984. [Form reflection of meteorological targets at the primary processing of the meteorological radar signal.] Trans. VGI 55. (In Russian).

Battan L. 1959. Radar meteorology. Univ. of Chicago Press, Chicago: 161 pp.

Chernikov A. 1979. [Radar clear sky echoes.] Hydrometeoizdat, Leningrad: 3-40. (In Russian).

Chernikov A., Shupyatsky A. 1967. [Polarization characteristics of radar clear sky echoes.] Trans. USSR Acad. Sci., Atmos. Ocean Physics 3, 2: 136-143. (In Russian).

Dinevich L. 2009. Radar monitoring of bird migration. Tel Aviv Univ.: pp. 1-173.

Dinevich L., Kapitalchuk I., Shupyatsky A. 1994. Use of the polarization selection of radar signals for remote sounding of clouds and precipitation. 34 ${ }^{\text {th }}$ Isr. A. Conf. Aerospace Sci.: pp. 273-277.

Dinevich L., Leshem Y., 2007. Algorithmic system for identifying bird radio-echo and plotting radar ornithological charts. Ring 29, 1-2: 3-40.

Dinevich L., Leshem Y., 2008. [Isolation of radar echo from migrating birds and plotting ornithological charts based on the data obtained by means of MRL-5.] Uspekhi sovremennoy radioelektroniki 3: 48-68. (In Russian). 
Dinevich L., Leshem Y. 2010. Radar monitoring of seasonal bird migration over central Israel. Ring 32, 1-2: 31-53. DOI: 10.2478/v10050-010-0003-z

Dinevich L., Leshem Y., Gal A., Garanin V., Kapitannikov A. 2000. Study of birds migration by means of the MRL-5 radar. J. Sci. Isr. - Technol. Adv. 2, 1: 94-104.

Dinevich L., Leshem Y., Matsyura A., 2005. Some characteristics of nocturnal bird migration in Israel according to radar monitoring. Ring 27, 2: 197-213.

Dinevich L., Leshem Y., Pinsky M., Sterkin A. 2004. Detecting birds and estimating their velocity vectors by means of MRL-5 meteorological radar. Ring 26, 2: 35-53.

Dinevich L., Leshem Y., Sikora O., 2001. Radar observations analysis of season bird migration in Israel at night (based on data of radar photo registration obtained in 1998-2000). J. Sci. Isr. Technol. Adv. 3, 1-2.

Dinevich L., Matsyura A., Leshem Y., 2003. Temporal characteristics of night bird migration above Central Israel - a radar study. Acta orn. 38, 2: 103-110.

Doviak R., Zrnic D. 1984. Doppler Radar and Weather Observation. Acad. Press: 512 pp.

Ganja I., Zubkov M., Kotjazi M. 1991. [Radar ornithology.] Stiinza, Kishinev: pp. 123-145. (In Russian).

Glover K., Hardy K. 1966. Dot angels: insects and birds. Proc. $12^{\text {th }}$ Weather Radar Conf. Am. Meteorol. Soc., Boston, 1966: pp. 264-268.

Hajovsky R., Deam A., La Grone A. 1966. Radar reflections from insects in the lower atmosphere. IEEE Trans. Antennas and Propagation 14, 2: 224-227. DOI: 10.1109/TAP.1966.1138665.

Houghton E. 1964. Detection, recognition and identification of birds on radar. World Conf. Radio Meteorol. Am. Meteorol. Soc., Boston: pp. 14-21.

Konrad T.G., Hicks J.J. 1966. Tracking of known bird species by radar. Proc. $12^{\text {th }}$ Weather Radar Conf. Am. Meteorol. Soc., Boston, 1966: 259-263.

Leshem Y., Dinevich L., Matsyura A. 2003. Studying Raptor Migration by a Network of Radar across Israel and Developing a Real Time Warming System for Flight Safety through the Internet. $6^{\text {th }}$ World Conf. Birds Prey Owls, Budapest, Hungary, 18-23 May, 2003

Richardson W.J., West T. 2005. Serious Birdstrike Accidents to U.K. Military Aircraft, 1923 to 2004: Numbers and Circumstances. IBSC 27 $7^{\text {th }}$ Meeting, Athens, Hellas, 23-27 May 2005: p. 5.

Rinehart R.F. 1966. Radar Detection of Birds in New Mexico. Preperint, Illinois State Water Surv.: p. 6.

Shestakova G. 1971. [The structure of bird wings and the mechanics of bird flight.] Moscow: 180 pp. (In Russian).

Shupyatsky A. 1959. [Radar dispersion of non-spherical particles.] Trans. CAO 30: 39-52. (In Russian).

Stepanenko V. 1973. [Radiolocation and meteorology.] Gidrometeoizdat, Leningrad. (In Russian).

Zavirukha V., Saricev V., Stepanenko V, Shepkin U. 1977. [Study of the dispersion characteristics of the meteorological and ornithological objects in echo-free cameras.] Proc. Main Geophys. Observatory 395: 40-45. (In Russian).

Zrnic D.S., Ryzhkov A.V. 1998. Observations of insects and birds with a polarimetric radar. IEEE Trans. Geosci. Remote Sensing 36, 2: 661-668. DOI: 10.1109/36.662746. 


\section{APPENDIX}

1) Receiver sensitivity $\boldsymbol{P}_{r}$ is the minimum power of a radar echo at the receiver entry that enables to obtain a nominal (set) value of the output voltage (at the given signal/noise ratio) that enables to steadily fix the signal against noise. Receiver sensitivity is usually measured in watts (W) and its values are within the range of $10^{-12}$ to $10^{-14} \mathrm{~W}$.

2) Radar beam $\theta$ characterizes the directional parameters of the radar antenna that affect the accuracy of determining target coordinates. The shape and the width of the beam may differ depending on the purpose of the station. The beam width of half power is the angle between directions in which the radiated power is $50 \%$ of the maximum power radiated along the beam's axis. The shape and the width of the beam depend on the type and dimensions of the antenna, as well as on the wave length $\lambda$.

3) Resolution capacity may be related either to the distance or to the coordinates:

a) Resolution over distance $r_{\min }$ is defined as the minimum distance between targets that are located along the same direction relative to the radar, at which those targets can be perceived by the radar as separate ones. In case the distance between the targets is below this minimum distance, the echoes of the targets merge on the radar screen. The resolution capacity over distance depends on the technical parameters of the equipment and the duration of the pulse within this space:

where:

$$
h=c \tau
$$

$c$ - the light speed

$\tau$ - the pulse duration (s).

b) Resolution over coordinates is defined as the minimum angular distance between targets located at the same distance, at which those targets can be perceived by the radar as separate ones. The resolution capacity over coordinates depends primarily on the radar beam width within the corresponding plane, as well as on the resolution capacity of the indicating equipment.

4) Radar distance - radio waves of length within the centimetre range propagate in the atmosphere within the line-of-sight, their trajectories being curvilinear rather than straight (the trajectory of radio-wave propagation is called a beam). This happens due to atmospheric refraction caused by the dielectric inhomogeneity of the atmosphere.

5) Radar equation for single targets - the radar equation relates the power of the echo with the technical parameters of the radar station, with the reflective properties of a target and the distance to it, as well as the conditions of radio-waves propagation on their way to the target.

6) Scattering cross-section (SCS or $\sigma$ ) of a bird - the reflective ability of a bird is determined by the dielectric properties, shape and dimensions of the bird body. It also depends on the length and polarization of the incident wave. 